\title{
Investigation of continuously adjustable extinction ratio in a multiwavelength SOA fiber laser based on intensity dependent transmission effect
}

\begin{abstract}
We investigate a flat multiwavelength fiber laser (MWFL) with the extinction ratio (ER) is continuously adjustable at variation of either half wave plate (HWP) position or polarization maintaining fiber (PMF) length. The multiwavelength laser is generated from an intensity dependent transmission (IDT) effect which is induced from nonlinear polarization rotation (NPR), arising from a combination of a semiconductor optical amplifier (SOA), polarization controllers (PCs) and polarization beam splitter (PBS). The ER is continuously adjustable from $6 \mathrm{~dB}$ to $12 \mathrm{~dB}$ by rotating the HWP of a PC, as the same value of the minimum and the maximum ER can be obtained in every $45^{\circ}$ of HWP position. The ER value is also adjustable at different length of PMF, as longer PMF is contributed to a flatter multiwavelength spectrum. The proposed MWFL design is simple, high number of lines, flat and able to adjust ER continuously.
\end{abstract}

Keyword: MuItiwavelength fiber laser; Lyot filter; Semiconductor optical amplifier; Intensity dependent transmission 